\title{
Educación en cultura, tecnología e innovación: una aproximación a los impactos del programa de formación de los Laboratorios Vivos de Innovación y Cultura*
}

Education on Culture, Technology, and Innovation: Labs Educational Program and its Impacts

Educação em cultura, tecnologia e inovação: uma abordagem aos impactos do programa de formação dos Laboratórios Vivos de Inovação e Cultura

\author{
Antonio José Ortega-Hoyos** (iD orcid.org/0000-0002-2985-7038 \\ Nathaly Gómez-Gómez*** iD orcid.org/0000-0002-6022-3893 \\ David Osorio-Vallejo**** (iD orcid.org/0000-0003-0199-7116
}

Para citar este artículo: Ortega-Hoyos, A. J., Gómez-Gómez, N., Osorio-Vallejo, D. (2020). Educación en cultura, tecnología e innovación: una aproximación a los impactos del programa de formación de los Laboratorios Vivos de Innovación y Cultura. Revista Colombiana de Educación, 1(80), 75-100. https://doi. org/10.17227/rce.num80-8279

$\begin{array}{ll}\text { (c) }(\text { ) } \$ & \text { Recibido: 06/08/2018 } \\ \text { Ev } & \text { Evaluado: 05/07/2019 }\end{array}$

* Este artículo se realizó en el marco del proyecto Laboratorios Vivos de Innovación y Cultura, implementado por la Fundación Universidad de Bogotá Jorge Tadeo Lozano, Seccional del Caribe en convenio con el Instituto de Cultura y Turismo de Bolívar (Icultur). Fue financiado por el Fondo de Ciencia, Tecnología e Innovación del Sistema General de Regalías, a través de la Gobernación de Bolívar (2015-2017)

** Magíster en gestión de la innovación. Estudiante de Doctorado en la Universidad de de Vigo, Pontevedra,

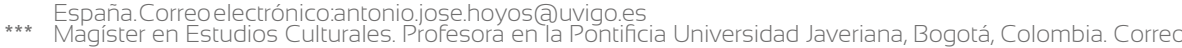
electrónico: nathaly.gomez@javeriana.edu.co

**** Especialista en Psicología del Consumidor. Estudiante de maestría en la Pontificia Universidad Javeriana; Bogotá, Colombia. Correo electrónico: david.osoriov@javeriana.edu.co. 


\section{Resumen}

En este artículo se exponen los principales impactos que se evidenciaron en los participantes del programa de formación desarrollado en el marco del proyecto Laboratorios Vivos de Innovación y Cultura en los municipios de Clemencia y María la Baja, en el departamento de Bolívar (Colombia). Para la investigación se utilizó una metodología cualitativa de tipo descriptivo-analítico con insumos como entrevistas, indicadores de seguimiento al proyecto y documentos internos que exponen la experiencia vivida en el desarrollo del proceso. Los impactos del programa se analizan a la luz de las capacidades humanas en relación con el objetivo del programa de formación, que buscó fortalecer las dimensiones cognoscitiva, psicomotoras y afectivas de los participantes. Entre los resultados, se encuentra que este programa contribuyó al estímulo de habilidades y capacidades sociales, al uso y la apropiación de las tic en relación con la generación de productos culturales, y a la consolidación de las identidades individuales y colectivas.

\section{Palabras clave}

desarrollo comunitario cognición; ciudadanía; habilidades; constructivismo; aprendizaje; educación en tecnología

\section{Keywords}

community development; cognition; citizenship; skills; constructivism; learning; technology education

\begin{abstract}
This paper presents the main impacts shown on participants in a cultural, technological, and innovational education program developed within the project Laboratorios Vivos de Innovación y Cultura [Living Labs for Innovation and Culture], in Clemencia and María la Baja, towns located in Bolívar, Colombia. A descriptive-analytical qualitative methodology was used. This implied the use of interviews, project follow-up indicators, and internal documents that condense the experience of being within the project. The education program impacts are analyzed through human capacities focused on the training program objectives, which sought to strengthen cognitive, psychomotor, and affective dimensions on the participants. It was found that the project contributed to building social skills and capacities, use and appropriation of Icts (Information and Communication Technologies), and consolidation of individual and collective identities.
\end{abstract}

\begin{abstract}
Resumo
Este artigo apresenta os principais impactos que foram evidenciados nos participantes do programa de capacitação desenvolvido no marco do projeto Laboratórios Vivos de Inovação e Cultura nos municípios de Clemencia e María la Baja, no departamento de Bolívar (Colômbia). Para a pesquisa, utilizou-se uma metodologia descritivo-analítica qualitativa com insumos como entrevistas, indicadores de monitoramento do projeto e documentos internos que expõem a experiência vivida no desenvolvimento do processo. Os impactos do programa são analisados à luz das capacidades humanas em relação ao objetivo do programa de formação, que procurou fortalecer as dimensões cognitiva, psicomotora e afetiva dos participantes. Dentre os resultados, constata-se que esse programa contribuiu para o estímulo de habilidades e capacidades sociais, para o uso e apropriação da Tıc em relação à geração de produtos culturais e para a consolidação de identidades individuais e coletivas.
\end{abstract}

\section{Palavras-chave}

desenvolvimento comunitário; cognição; cidadania; competências; construtivismo; aprendizagem; educação tecnológica 


\section{Introducción}

El panorama general de la educación tiene pluralidad de influjos teóricos y prácticos provenientes de diversas corrientes pedagógicas. Una de ellas es el constructivismo, cuyos conceptos centrales son el aprendizaje significativo y el experiencial. Con estos como eje, el programa de formación del proyecto Laboratorios Vivos de Innovación y Cultura, ${ }^{1}$ en adelante Laboratorios Vivos, le apostó a la generación de "una estrategia de formación, mediación y emprendimiento cultural para la apropiación social del conocimiento y la generación de innovación social en las comunidades de Clemencia y María la Baja" (Gobernación de Bolívar, Icultur y Utadeo, 2014, p. 44). Aquí se relacionan algunos de sus impactos en los participantes del programa.

La implementación del programa de formación ${ }^{2}$ tuvo diferentes momentos clave. En la figura 1 se presenta la línea de tiempo de las fases del programa, usando los meses de ejecución del proyecto como referente.

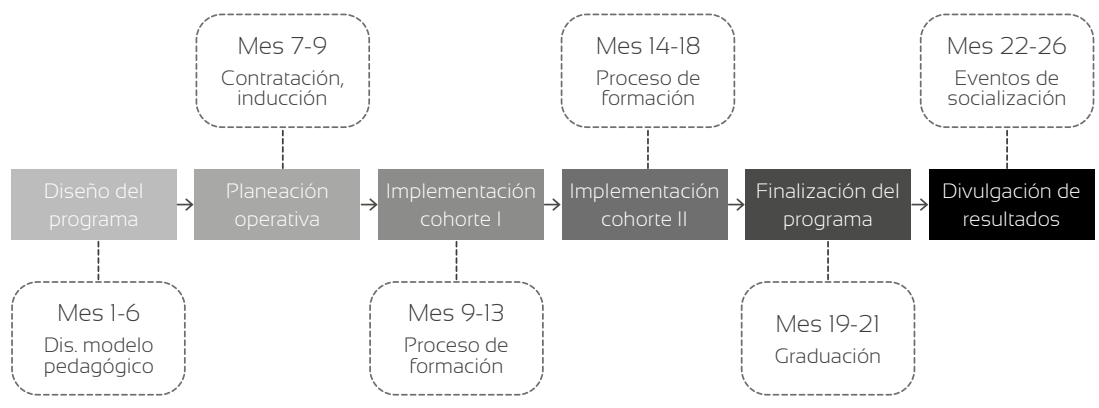

Figura 1. Fases del programa de formación de los Laboratorios Vivos de Innovación y Cultura Fuente: elaboración propia con base en el área de formación.

Nota. Los meses señalados corresponden al periodo en que se ejecutó el proyecto, desde julio del 2015 (mes 1) hasta octubre del 2017 (mes 26).

El programa educativo estuvo enmarcado en la formación no formal y tuvo como conceptos transversales la cultura, la tecnología y la innovación social en dos cohortes ${ }^{3}$ con participantes de cuatro perfiles (véase figura 2).

1 Inicialmente conocido como "Implementación de una estrategia para el uso y apropiación de la cultura como generadora de conocimiento e innovación social, a través de laboratorios sociales de investigación y creación en el departamento de Bolívar".

2 Para conocer detalles del diseño del programa puede ampliar información en el documento Manual proyecto educativo del Programa de Formación con Enfoque de Innovación Social". En él se consolidan los conceptos, estrategias pedagógicas y metodológicas que se tuvieron en cuenta en el proceso de formación.

3 La primera cohorte se realizó entre mayo y septiembre del 2016, y la segunda, entre septiembre del 2016 y febrero del 2017. 
El centro de operación del programa fue la Casa de la Cultura de cada municipio, con una dotación de equipos tecnológicos ${ }^{4}$ y la adecuación infraestructural de espacios para albergar dicha tecnología.
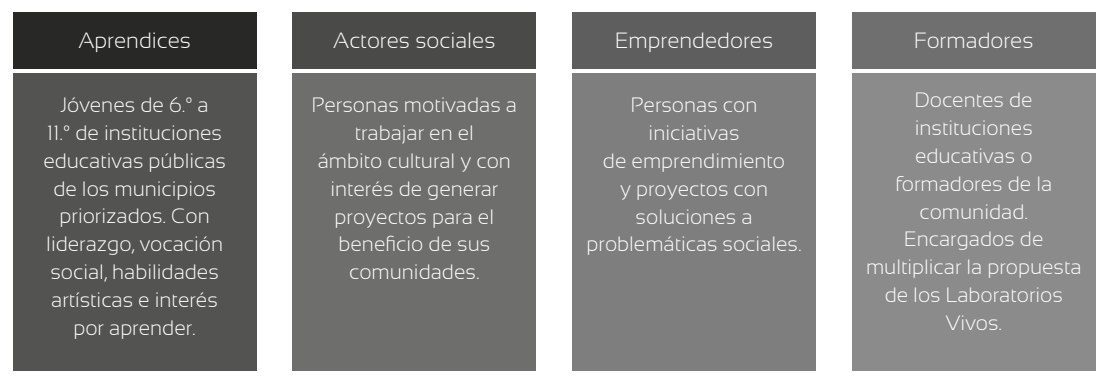

Figura 2. Perfiles del programa de formación.

Fuente: elaboración propia con base en datos del área de formación.

Los participantes de cada uno de los cuatro perfiles cursaron de dos a cinco módulos en el programa de formación, en el siguiente orden: 1) Usos y apropiación de la cultura, 2) Procesos de investigación-creación, 3) Gestión y emprendimiento, 4) TIC y la producción audiovisual, y 5) Formación, cultura y generación de conocimiento, bajo modalidad presencial. El único perfil que cursó todos los módulos fue el de Formadores, dada la intención de multiplicar el conocimiento.

Desde un enfoque de investigación-creación, ${ }^{5}$ los participantes realizaron actividades que promovieron la indagación y resolución de problemas, apoyados en las tecnologías de la información y las comunicaciones ${ }^{6}$ (TIC) como herramientas de mediación de nuevas formas de investigación y de generación de emprendimientos culturales. Para promover el uso y la apropiación de la cultura utilizaron estrategias pedagógicas como la realización

4 Cámaras, computadores, tablets, tableros digitales, luces (halógenas, fluorescentes y de tungsteno), pantallas reflectoras trípodes, mezclador, micrófonos, entre otros.

5 La metodología central del programa de formación fue la investigación-creación apoyada en el enfoque del aprendizaje basado en la investigación, el cual se orienta a "hacer uso de estrategias de aprendizaje activo para desarrollar en el estudiante competencias que le permitan realizar una investigación creativa en el mundo del conocimiento" (Torres, 2006, p. 2). Es una manera didáctica de articular la enseñanza con la investigación y brinda la oportunidad de cultivar el espíritu investigativo en los participantes, como una estrategia para producir conocimiento contextual y pertinente (Utadeo, 2016, p. 44).

6 Según la ley colombiana, las TIC son "el conjunto de recursos, herramientas, equipos, programas informáticos, aplicaciones, redes y medios, que permiten la compilación, procesamiento, almacenamiento, transmisión de información como: voz, datos, texto, video e imágenes" (Ley 1341 de 2009, artículo 6). 
de los microproyectos, ${ }^{7}$ el uso de las TIC $^{8}$ y las actividades de popularización, ${ }^{9}$ por nombrar algunas. Esto bajo el liderazgo de docentes-mediadores y técnicos especializados en las áreas de sistemas, video y audio. En la figura 3 se presentan los principales datos del programa de formación.

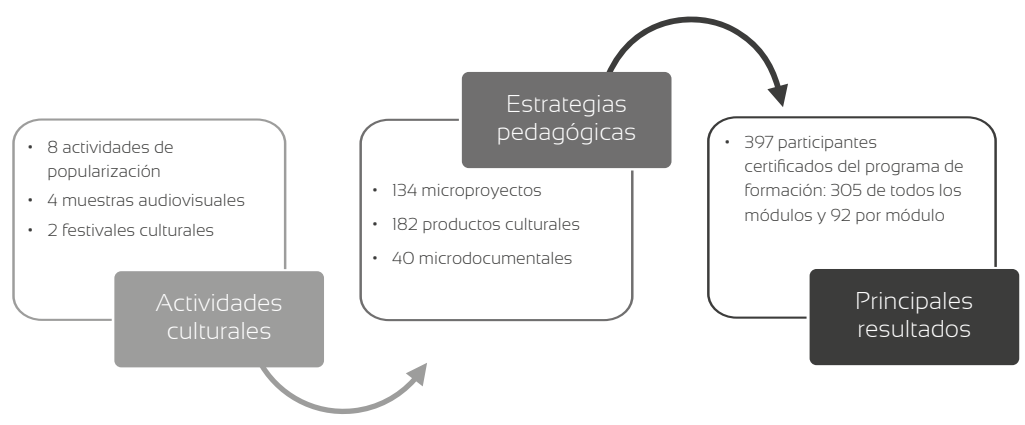

Figura 3. Principales datos del programa de formación de los Laboratorios Vivos

Fuente: elaboración propia con base en el área de formación.

\section{Fundamentos teórico-conceptuales}

[El constructivismo] como corriente pedagógica contemporánea, representa quizá la síntesis más elaborada de la Pedagogía del siglo xx, porque constituye una aproximación integral de un movimiento histórico y cultural de mayores dimensiones: la Escuela Activa. Movimiento que en su tiempo asumió una concepción reformista y una actitud transformadora de los procesos escolares" (Miranda, 2004, p. 1).

7 Fueron concebidos como las iniciativas de innovación ideadas por los participantes del proyecto, que se perfeccionaron en su paso por los módulos del proceso de formación, los cuales generaron productos de innovación con potencia de emprendimientos culturales (Utadeo, 2016, p. 54).

8 Se concibió como una estrategia pedagógica vertebral del programa de formación, por su carácter transversal a los procesos de apropiación social del conocimiento, aprendizaje e innovación social. Coherente con la metodología de investigación-creación, los Laboratorios Vivos de Innovación y Cultura le apuntan al fortalecimiento de las capacidades y habilidades de los participantes de los municipios de Clemencia y María la Baja en torno al uso pedagógico de las TIC para la generación activa de conocimiento (Utadeo, 2016).

9 Las actividades de popularización se desarrollaron mediante demostraciones, dispositivos interactivos, socializaciones y el uso de medios alternativos, donde los participantes dieron cuenta del proceso formativo, involucrando a sus coterráneos, como una forma de generar apropiación social del conocimiento (Utadeo, 2016, p. 60). 
Influenciado por autores como Piaget, tuvo su origen "en la revolución cognoscitiva de los años setenta, para enfrentar la insatisfacción dejada por el paradigma del aprendizaje, hasta entonces dominante: la psicología conductista y el asociacionismo" (Almeida, 2005, p. 1). Los principios epistemológicos del constructivismo son

el conocimiento no es una copia de la realidad sino una construcción del ser humano, existen múltiples realidades construidas individualmente y no gobernadas por leyes naturales y la ciencia no descubre realidades ya hechas, sino que construye o crea realidades. (Almeida, 2005, pp. 2-3)

A partir de los retos de las sociedades contemporáneas, la educación desde esta mirada constructivista busca construir conocimiento con la participación de los estudiantes, sus trayectorias y experiencias. El constructivismo aparece, desde la mirada de Solé y Coll (1997), como una herramienta y un marco explicativo que da cuenta de la función social y socializadora que tiene la educación en la formación de individuos que están inmersos en un contexto cultural que no les es ajeno. Este modelo no busca

[un] aprendizaje de un contenido sino el desarrollo y afianzamiento de las estructuras mentales del conocer y del aprender. Se trata no tanto de memorizar contenidos sino de involucrarse en un proceso dinámico de conocimiento que desarrolle las destrezas cognoscitivas mediante modelos de descubrimiento y solución de problemas. (Ramírez, 1999, p. 4)

Por el contrario, se buscaba desarrollar pensamiento autónomo y creativo para que los participantes entendieran significativamente su mundo.

El constructivismo se apoya en la estructura conceptual del estudiante y procura una construcción activa del nuevo concepto, aplica el nuevo concepto a situaciones concretas y lo relaciona con otros conceptos para ampliar su transferencia (Ramírez, 1999). En esta misma línea, el aprendizaje significativo plantea que la estructura cognoscitiva previa de la persona, entendida como las ideas y los conceptos que se poseen en un campo del conocimiento y su organización, se relaciona con nueva información. Esto quiere decir que existe un vínculo entre los conocimientos previos y los nuevos, y es a partir de esta relación que se construye el aprendizaje (Ausubel, 2002).

El conocimiento antiguo sirve como sustrato para que el nuevo se ancle de forma no arbitraria y sustantiva; para esto, el conocimiento anterior debe estar consolidado, claro y disponible en el sujeto. Al lograr un aprendizaje significativo, se consigue construir significado, dar sentido a lo aprendido, y entender los diferentes espectros de aplicación 
y la relevancia en situaciones académicas y cotidianas. Ambos conocimientos, el nuevo y el antiguo, van reconstruyéndose y reajustándose (Ausubel, 1983; 2002).

Para que este proceso sea significativo se requiere una agencia del estudiante, derivada principalmente de su motivación hacia un tema específico del que derivaría gran parte de su trabajo dentro de los laboratorios. Por esta razón, la búsqueda y hallazgo de lo que involucra emocionalmente al estudiante se presenta como eje fundamental a la hora de estimular y potenciar el aprendizaje, ya que las emociones son cómplices críticas del pensamiento y la racionalidad. Al priorizar temas de relevancia personal, elaborar memorias e influir en la toma de decisiones, impregnan la actividad individual y colectiva (Darder et al., 2017).

Dentro del programa se promovió un cambio en los roles que existen convencionalmente en la educación: profesor y alumno. Para el contexto del programa de formación, a estos se les referenció como mediador y participante, dado que la apuesta era lograr articular los conocimientos de las dos partes a partir de sus acercamientos al tema, trayectorias y experiencias. Se pretendía que el participante construyera nuevo conocimiento en una experiencia de aprendizaje significativo para producir iniciativas de innovación social a través de la investigación-creación y la apropiación social del conocimiento.

El aprendizaje redunda en desarrollo humano, en consonancia con la perspectiva del Programa de las Naciones Unidas para el Desarrollo (PNUD), que considera que el bienestar se mide más allá del ingreso y consiste en la ampliación de las opciones de las personas. Por lo anterior, se trata de un enfoque en el cual se privilegia la capacidad con que cuentan las personas en su entorno para acceder efectivamente a bienes y servicios que les permitan ser y hacer en ejercicio de su libertad (PNUD, 2016).

El desarrollo humano es un enfoque multidimensional que trasciende las esferas económicas para incluir elementos de la vida social, psicológica, educativa, del bienestar físico, entre otras. Este enfoque propone la generación de capacidades en los individuos, y establece que el desarrollo de estos es posible en la medida en que se provean las oportunidades para materializar sus derechos a través del ejercicio de sus libertades. Es decir, que las personas cuenten con capacidades para ser o hacer lo que deseen en un contexto y momento histórico determinado (Sen, 2004).

Por su parte, se entiende que los impactos están "compuesto[s] por los efectos a mediano y largo plazo que tiene un proyecto para la población objetivo y para el entorno, sean estos efectos o consecuencias deseadas (planificadas) o sean no deseadas" (Bello, 2009, p. 3). En esta investigación los impactos se entienden como los efectos inmediatos durante el proceso de formación manifestados por los participantes. 
Para aproximarse a los impactos, se indagó acerca de las teorías y los conceptos desde los cuales se dio origen al plan de estudios y la visión por desarrollar en el programa de formación de los Laboratorios Vivos. La estrategia de enseñanza y aprendizaje se enmarcó en los objetivos del programa de formación descritos como dominios:

\section{Dominio cognoscitivo}

Hace referencia al aprender a conocer.

Al tomar como punto de partida el conocimiento adquirido se pueden construir nuevas relaciones en el conocimiento, al mismo tiempo que se alcanza la comprensión de conceptos, terminologías, para normalizar conceptos y hablar el mismo idioma, hechos y acontecimientos, creencias, costumbres de cada localidad, entendidos como fenómenos sociales y culturales de interés. (Utadeo, 2016, p. 70)

\section{Dominio psicomotor}

Se refiere al aprender a hacer: "que garanticen el manejo técnico adecuado de los equipos tecnológicos visuales y audiovisuales (cámaras de video, micrófonos, software de edición audiovisual, entre otros), como herramientas que contribuyen a la generación de productos de innovación" (Utadeo, 2016, p. 69).

\section{Dominio afectivo}

Se relaciona con el aprender a ser:

Cada beneficiario debe ser capaz de reconocerse a sí mismo en el contexto del municipio y de esta manera ser capaz de reconocerse en el otro. En el reconocimiento de la identidad local, es determinante aceptarse en la interacción social y en la diversidad cultural que caracteriza cada uno de estos lugares. (Utadeo, 2016, p. 71)

La combinación del constructivismo, el desarrollo humano, el aprendizaje significativo y los dominios permitió que surgiera un programa de formación integral. Este buscó no solo impartir, integrar y construir conocimiento, sino también contribuir a otras esferas de la vida del participante para que este pudiese aplicar lo aprendido en su vida personal, social, profesional y comunitaria. 


\section{Metodología}

La investigación es de corte cualitativo, descriptivo-analítico. La mayoría de la información empleada es cualitativa, con excepción de la inclusión de indicadores de seguimiento del proyecto. ${ }^{10} \mathrm{El}$ enfoque al que se adscribe la investigación es el construccionismo social (Gergen, 1996); ; se usa el análisis del discurso para develar los sentidos alrededor de los impactos del programa de formación de los Laboratorios Vivos. Se entiende una relación bidireccional entre el lenguaje-discurso y la práctica como hechos sociales. Así, el discurso se transforma en prácticas y estas desembocan en discurso. Las técnicas para obtener la información fueron entrevistas a profundidad ${ }^{12}$ individuales (23) realizadas a participantes de las dos cohortes, y entrevistas individuales y grupales (3) a 6 miembros del equipo del programa de formación. Se realizó una entrevista individual, una diada y una triada. Se revisaron 5 indicadores y 10 documentos sobre la sistematización de la experiencia de los mediadores. Además, como fuente de información se tuvieron en cuenta documentos que exponen la experiencia del proyecto elaborados por el equipo de formación. ${ }^{13}$

Se formularon dos cuestionarios, uno para los participantes del proyecto y otro para los miembros del equipo de formación; el último se utilizó en las entrevistas, diadas y triadas. El objetivo de los instrumentos era aproximarse a la experiencia en el programa de los participantes e integrantes del área de formación. La información se analizó a la luz de tres categorías: Conocer, Ser y Hacer, las cuales corresponden a cada uno de los tres objetivos del programa de formación.

10 Es el conjunto de datos, agrupados por los componentes del proyecto que se medían y analizaban mensualmente. Contiene la línea de base y seguimiento desde el año cero del proyecto, describe los indicadores diseñados, sus fórmulas de cálculo, fuentes de verificación y metas, que son insumos para hacer el seguimiento y medir el impacto del proyecto en los municipios intervenidos.

11 En el sentido de analizar y deconstruir los sistemas de significados producidos a partir del relacionamiento y la coordinación entre individuos.

12 Las entrevistas a profundidad a los participantes del programa de formación fueron diseñadas originalmente como un instrumento para el documento de sistematización y análisis de entrevistas a profundidad del área de investigación. Se realizaron en los municipios, y tuvieron como principal objetivo describir y analizar la forma en la cual se generó la apropiación social del conocimiento y usos de la cultura en términos de innovación social en el marco del programa de formación. Estas se realizaron entre enero del 2016 y marzo del 2017. Agradecemos el apoyo a los coinvestigadores Federico Ochoa, Laura Mendoza y Antonio Ortega, y a los auxiliares de investigación Kimberly Marín y Miller García en la realización de las transcripciones de las entrevistas.

13 El equipo de formación estuvo conformado por un jefe, una coordinadora municipal y una coordinadora de formación por territorio, y tres técnicos en cada municipio (encargados de cada una de las salas: video, audio, sistemas y sonoteca). También contó con los mediadores, quienes son fuente de información a partir de sus informes de experiencia en las sesiones con los participantes y tuvieron a cargo el desarrollo de los módulos señalados. Agradecemos a los miembros del equipo de formación por el apoyo y la información brindada para esta investigación. 


\section{Resultados}

\section{Impactos en la dimensión cognoscitiva}

El dominio cognoscitivo se relacionó con aprender a conocer. En este sentido, como manifiesta Chaparro (2001)

para que el conocimiento pueda convertirse en factor de crecimiento y de progreso, es necesario ir más allá del desarrollo de una capacidad para generarlo. Se requiere que este último sea interiorizado por parte de individuos, organizaciones, empresas e instituciones sociales, es decir, que el conocimiento permée la sociedad. (p. 23)

Cada módulo del programa de formación se constituyó en una pieza clave para la generación de capacidades en torno al uso y la apropiación de la cultura como medio para generar procesos de innovación social, transversalizados por el uso de las TIC, en pro del desarrollo de las comunidades. Para evaluar el nivel de conocimiento de los participantes acerca de los contenidos de los módulos, se realizó una prueba diagnóstica al iniciar y una prueba de salida al finalizar, cada una de estas buscaba dar cuenta de los conocimientos específicos de las temáticas abordadas. Estas pruebas de entrada y salida contenían preguntas relacionadas con cultura, principios de la investigación-creación, emprendimiento, elementos de la producción audiovisual, y herramientas para la formación y el trabajo en comunidad.

De acuerdo con los resultados de las pruebas, en la primera cohorte del programa en Clemencia, en el 33,7 \% de las evaluaciones de salida se obtuvieron mejores puntajes que al inicio de los módulos. En esta misma cohorte, en María la Baja este resultado correspondió al 32,1\%. Se observó que en la segunda cohorte del programa de formación estos resultados se ubicaron en un $66,6 \%$ para Clemencia y en un $43,4 \%$ para María la Baja. El análisis de los resultados permitió identificar el nivel de los conocimientos previos, las principales deficiencias en conceptos, al igual que la apropiación del conocimiento que lograron los participantes al finalizar el programa de formación.

Se evidenciaron cambios positivos en los aprendizajes obtenidos, que dan cuenta de una apropiación de los contenidos desarrollados. Es necesario resaltar que en la primera cohorte en Clemencia el 64,5 \% de las evaluaciones de salida presentadas obtuvieron una nota aprobatoria; es decir, una nota igual o superior a 6 sobre 10. Por su parte, en María la Baja este porcentaje estuvo en el 78,5 \%. En la segunda cohorte el 92,5\% de las evaluaciones tuvieron notas aprobatorias en Clemencia y 88,9\% en María la Baja, lo que quiere decir, un porcentaje mayor de las evaluaciones en la segunda cohorte obtuvieron una nota por encima de 6. 
La información recogida, además de reafirmar los procesos adelantados en los módulos desarrollados, arrojó varios impactos relacionados con los conocimientos adquiridos por los participantes entrevistados. Se evidencia que, de una u otra forma, tanto en Clemencia como en María la Baja, la mayoría de los participantes lograron procesos de identificación de las principales teorías, herramientas y técnicas para aplicarlos de manera práctica en sus trabajos y en su vida cotidiana. Fundamentalmente aprendieron sobre aspectos sociales y culturales.

Los participantes aprendieron de las manifestaciones culturales de sus municipios como mecanismo para lograr una mayor identidad cultural en el módulo de Usos y Apropiación de la Cultura; del manejo de técnicas de investigación-creación en el módulo con el mismo nombre; de instrumentos de planeación y formulación de modelos de negocios en el módulo de Gestión y Emprendimiento; del manejo de herramientas audiovisuales en el módulo de TIC y la Producción Audiovisual; y de apropiación y gestión del conocimiento en el último módulo de Formación, Cultura y Generación de Conocimiento. Un resumen y ejemplo práctico de esto lo ofrece la docente Fermina Villamil, perteneciente al perfil Formadores en María la Baja:

En el primer módulo de usos y apropiación de la cultura, que fue con Nemecio, ${ }^{14}$ todo lo que tenía que ver con cultura, ya en el de investigación-creación fueron los inicios de la elaboración del microproyecto. Y en el de emprendimiento, aprendí a elaborar un Canvas, que yo no sabía, y con David ${ }^{15}$ que he aprendido todo el manejo de equipos, no soy una experta, pero al menos me atrevo a realizar una grabación o tomar unas fotografías, claro que para ser una experta uno tiene que llegar mucho más allá; y ahora en el último módulo, que vimos la semana pasada con Luz, ${ }^{16}$ que ha sido como un engranaje de todo lo que hemos visto y de alguna manera cómo nosotros proyectamos todo lo que hemos visto a nuestra actividad pedagógica. (Fermina Villamil, María la Baja, entrevista, 13 de febrero del 2017)

Algunos de los participantes entrevistados evidenciaron un mayor reconocimiento y valoración de sus manifestaciones culturales en torno a las prácticas cotidianas y la construcción de la identidad local de su municipio relacionado con la música, las décimas y los concursos de canarios. Al respecto, Dulzania Salcedo, del perfil Actor social, en Clemencia, mencionó: "bueno, yo no sabía antes que había un concurso de canario, yo tengo

14 Nemecio Berrío, mediador del módulo de Investigación-Creación del Laboratorio Vivo de María la Baja.

15 David Covo, mediador del módulo de TIC y Producción Audiovisual del Laboratorio Vivo de María la Baja.

16 Luz del Carmen Gloria, mediadora del módulo de Formación, Cultura y Generación de Conocimiento, que adelantó su proceso de mediación en los dos municipios. 
unos añitos viviendo acá, pero no sabía que se daba aquí los concursos de canarios. Bueno varios concursos aquí que no sabía que los hacían" (Dulzania Salcedo, Clemencia, entrevista, 15 de julio del 2016).

Otros participantes entrevistados manifestaron haber aprendido de forma dinámica el manejo de herramientas útiles para su vida, lo cual valoran y aprecian; por ejemplo, la señora Luz Barrios, formadora de Clemencia, es consciente de las técnicas empleadas en el proceso de formación, parece comprenderlas y les ve utilidad. Así lo expresó: "[Vimos] cómo nosotros con la cultura que tenemos, podemos innovar hacia otra cultura que nos lleve a un aprendizaje más significativo" (Luz Barrios, Clemencia, entrevista, 8 de septiembre del 2016).

En cuanto a los aprendizajes más prácticos se encuentran las diferentes técnicas e instrumentos para la formulación de modelos de negocios aprendidos por los participantes en el módulo de Emprendimiento. Al respecto, Luz Barrios expresó lo siguiente:

Bueno, con la seño Alexandra, ella tuvo una forma tan lúdica y participativa de hacer los talleres [...]. Por cada contenido, ella nos daba un dibujo, unas imágenes, o sea, ella ayudaba a que uno desarrollara el pensamiento más rápido y que las cosas le quedaran a uno, y no se le olvidara. Por ejemplo, yo no puedo olvidar de lo que nos dio, el modelo Canvas, una estructura de negocio que me quedó, por lo que yo creo que estoy presta a poner un negocio con ese modelo. (Luz Barrios, Clemencia, entrevista, 8 de septiembre del 2016)

En este mismo sentido, la participante Sol Rodríguez, del perfil Emprendedores, de María la Baja, expresó lo que ha sido más gratificante para ella del proceso de formación de los Laboratorios Vivos, haciendo énfasis en la oportunidad que visiona con el programa e incluso tratando de aplicar a sus ideas de emprendimiento:

Bueno, al principio yo decía, aquí voy a ayudar a muchos, es algo chévere, una experiencia que en verdad yo no pensé que iba a adquirir tanto conocimiento y no solamente teórico sino práctico. Uno va adquiriendo unas experiencias y unos conocimientos que le van a quedar de por vida, es algo que me he dado cuenta. [En las] universidades o en los colegios no se adquiere esos conocimientos que en verdad hemos adquirido aquí, ya que no solamente rescatamos nuestras habilidades sino también nuestra cultura para la vejez, o sea, eso fue algo más que impactante porque en verdad había cosas que uno como que "ah" [...]. De resaltar de pronto lo que es el bullerengue o nuestro tesoro cultural, como yo digo que es María la Baja, nuestra fauna, nuestra flora y nuestra cultura, entonces, eso fue lo que más me impactó y me ha quedado, es más, que el proyecto mío lo cambié, o sea, yo venía con una mentalidad de yo voy a coger, voy a tejer y voy a trabajar con la comunidad tejiendo, enseñando a tejer. Cuando Ilegué aquí a medida 
que iban pasando todas las clases [...], pero si podemos hacer esto, podemos trabajar con la comunidad, pero también resaltando más que todo la cultura y todo eso [...], incluyo no solamente mi proyecto como tal sino los proyectos de todos los que están aquí en los Laboratorios.

(Sol Rodríguez, María la Baja, entrevista, 31 de agosto del 2016)

En estos testimonios de los participantes se evidencia cómo se han ido transformando sus pensamientos gracias a las nuevas formas de aprendizajes para trabajar sus prácticas culturales. A través de los procesos adelantados en cada temática se ha contribuido a la recuperación, valoración y apropiación de la cultura por parte de los participantes de las comunidades de Clemencia y María la Baja.

\section{Impactos en la dimensión psicomotora}

Este dominio dedicado al aprender a hacer se centra en el fomento de habilidades físicas y psicológicas desde los primeros años de infancia con el objetivo de que la persona tenga unas destrezas relacionadas con la coordinación, así como con su capacidad de adaptabilidad y cualidades para expresarse e interpretar (Harrow, 1978). Asimismo, existe una taxonomía en la que se hace

énfasis en las destrezas motoras (escribir), hasta aquellas que son altamente integradas con logros cognoscitivos y afectivos (ejecutar un experimento). La clave está en concentrarse en lo que los estudiantes van a hacer, no en lo que van a decir: en los procesos, más que en los productos. (Simpson, 1972, citado en Medina y Verdejo, 2001, p. 52)

En el caso del programa de formación de los Laboratorios Vivos, este dominio estuvo relacionado con "aquellos conocimientos de tipo procedimental [...] que garantizaran el manejo técnico adecuado de los equipos tecnológicos visuales y audiovisuales (cámaras de video, micrófonos, software de edición audiovisual, entre otros), como herramientas que contribuyen a la generación de productos de innovación" (Utadeo, 2016, p. 69). Para poder analizar el impacto que tuvieron los participantes del programa de formación se debe tener en cuenta en qué medida ellos reconocían y usaban los diferentes espacios y equipos de las casas de la cultura de los municipios y cómo elaboraban los diferentes productos culturales.

Al revisar la batería de indicadores de seguimiento del proyecto, se hallaron los siguientes datos relacionados con las visitas de los participantes a los diferentes espacios dispuestos para ellos, y su uso para la creación de contenidos culturales mediante el manejo de las herramientas digitales disponibles: un total de visitas a salas de 3336 en Clemencia, y 1094 en María la Baja. 
La apropiación de los espacios por parte de participantes y comunidad en general fue más evidente en Clemencia. Esto se enmarcó en el desarrollo de actividades culturales en las cuales las TIC fueron parte fundamental del proceso. La sala de sistemas de Clemencia fue el espacio con mayor número de visitantes (en promedio más de 176 visitas mensuales), el uso de computadores e internet fue dedicado principalmente a actividades de entretenimiento y búsqueda de información. La apropiación del espacio y los equipos se hizo más evidente a medida que pasó el tiempo, lo que posibilitó un acercamiento a las nuevas TIC, que permitió conectar a los municipios con el mundo, más aún teniendo en cuenta el bajo índice de penetración de internet en el municipio, 1,1 \% (Ministerio de Tecnologías de la Información y las Comunicaciones, citado en Osorio y García, 2017).

En el caso de María la Baja, la sala de audio fue la que tuvo mayor número de visitas (en promedio 52 mensuales), situación que se relaciona con los talleres de técnica vocal impartidos por la profesional de audio Lizeth Núñez. Estos talleres tuvieron tan buena acogida por parte de la comunidad, que se creó un programa denominado Voces Vivas, que contó con la participación de algunos beneficiarios del proyecto.

$\mathrm{Al}$ analizar en las entrevistas a profundidad los primeros contactos que tuvieron los participantes con las salas de audio, video, sistemas y sonoteca, se observó que la gran mayoría estuvieron relacionados con los módulos que se llevaban a cabo, con los recorridos de las primeras sesiones por los espacios de las casas de la cultura y con la creación de los productos y las diferentes actividades del proyecto, como las actividades de popularización y muestras audiovisuales. Las siguientes citas lo ejemplifican.

La formadora Luz Barrios comentó cómo fue la dinámica de aprendizaje de las clases y su acercamiento a los equipos disponibles:

Primero la teoría y después la práctica. Nos explicaban, por decir algo, cómo coger el micrófono, cómo utilizar las cámaras, cómo utilizar los computadores, y las clases eran compartidas, primero el profesor partía de la experiencia que uno tenía, y de ahí ellos construían después un nuevo conocimiento y siempre nos hacían clase hecha, clase evaluada. (Luz Barrios, Clemencia, entrevista, 8 de septiembre del 2016)

Con las sesiones dedicadas al conocimiento y buen uso de los equipos tecnológicos disponibles es posible ver cómo se desplegaron procesos que influyeron en los participantes, quienes, a pesar de no tener muchas bases sobre el tema, lograron incorporarlos a su vida diaria. Frente a esto, Fermina Villamil, formadora de María la Baja, relató como lo que aprendió en este proceso lo ha ido transmitiendo en sus espacios 
cotidianos y ha llegado a desarrollar una "fiebre"17 por el proyecto, como parte del efecto que ha tenido en ella conocer más de su cultura y el uso de las herramientas TIC:

Yo creo que día a día voy expresando en la casa todo lo que hago aquí, todo lo que he aprendido aquí, desde la valoración de mi cultura hasta el manejo de equipos audiovisuales, cámaras fotográficas, manejo de TIC, o sea yo aquí he aprendido muchas cosas..., porque yo no soy una nativa digital, pero siempre estoy en ese proceso constante, mejor dicho, que a mí me dicen que yo tengo fiebre con los Laboratorios Vivos, yo todos los días llego aquí. (Fermina Villamil, María la Baja, entrevista, 13 de febrero del 2017)

En el caso del aprendiz marialabajense Arnulfo Saldarriaga, este aprendió en el módulo de TIC y producción audiovisual no solo a manejar los equipos disponibles en la Casa de la Cultura, sino también a utilizar su teléfono celular como herramienta que le permite practicar contenidos de la clase, por ejemplo a realizar efectos fotográficos de una silueta, tal como se puede leer a continuación:

El profesor del módulo de TIC [...] mientras nos enseñaba, también nos mostraba cosas para que nosotros entendiéramos, también aprendimos que se podían hacer con el teléfono unas formas al espacio de la silueta del cuerpo y no salía la persona que le estaba haciendo la silueta a la otra persona, ahí nos enseñaba varias cosas, o jugábamos y mientras jugábamos él iba explicando la clase. (Arnulfo Saldarriaga, María la Baja, entrevista, 13 de febrero del 2017)

Una de las grandes dificultades que se presentaron durante este proceso tuvo que ver con que varios de los participantes (particularmente de los perfiles de formadores, actores sociales y emprendedores) por su edad, no contaban con los conocimientos previos sobre el uso de tecnologías como el computador, lo cual dificultó el desarrollo de las primeras sesiones dadas por los mediadores y que incluían actividades que requerían el uso de estas tecnologías.

Para mejorar los conocimientos de los participantes sobre las herramientas tecnológicas y su aplicación en la creación de contenidos culturales, hay que tener en cuenta también la pertinencia del desarrollo de las temáticas, porque, por ejemplo, el módulo de TIC y producción audiovisual ${ }^{18}$ solo lo vieron formadores y aprendices, es decir que los

17 Este síntoma de "fiebre" puede haberse generado por el germen simbólico-tecnológico que ha propagado los Laboratorios Vivos en los municipios. Para conocer mejor sobre esto dirigirse a la introducción del libro "Tecnologías simbólicas y culturas creativas. La experiencia regional de los Laboratorios Vivos" (Molina, Zúñiga, Mendoza y Marín, 2017).

18 Este módulo es el que más referencian los participantes cuando se les pregunta por el uso y aprendizaje del manejo de los equipos tecnológicos y la creación de contenidos. 
demás perfiles de formación no gozaron de esta oportunidad. De igual forma, también es importante la designación de los tiempos y enfoques para producir algo en estas salas, que en ocasiones va en contravía de lo que realmente se quería hacer. El emprendedor de María la Baja, Elkin Fuentes, lo relató así:

En la sala de video, se les dice a las personas que se les va a hacer una producción. Se les da un turno para que lo hagan, pero el tiempo no era suficiente para que ellos cumplieran sus necesidades personales. Se tiene como prioridad a los participantes del Laboratorio, pero [estos] quieren otras cosas, por ejemplo, Sobrecallo ${ }^{19}$ participa y adquiere también un video de grabación, pero el espacio que se le da es muy corto, entonces, es como un poquito frustrante para otras personas. No hay un tiempo especificado para que todos puedan beneficiarse de esto.

En el estudio de grabación fue chévere, pero las producciones seguían más a la producción de radio y no a la producción musical, es decir, grabar una canción ahí es muy difícil, no hay monitores, no hay retorno donde se pueda hacer una buena mezcla de grabación, había uno solo, pero se dañó y no se usó más. Entonces, yo entré con las ganas, pero eso me desmotivó bastante, porque cómo voy a grabar y después que grabe cómo voy a mezclar y después, no vi como que las herramientas para hacer una producción musical como tal. Entonces, se aparta turnos para que los muchachos graben canciones y todo, pero la calidad, a pesar de que es un estudio que tiene buenos equipos, no cumple con la necesidad de los que acceden a él. (Elkin Fuentes, María la Baja, entrevista, $1{ }^{\circ}$ de septiembre del 2016)

Uno de los puntos por mejorar fue no concebir en un comienzo una alfabetización tecnológica en los participantes; además, no hubo un suficiente fomento del uso de los espacios, más allá de las tareas o productos presentados en el marco del proyecto. A pesar de esto, la mayoría de los entrevistados reconocen cuáles son las salas y algunos de los elementos que se encuentran allí, con lenguaje técnico o sin él, y además pudieron desarrollar habilidades para su uso que aplican en su vida diaria. Esto da pistas de a qué le apunta el dominio psicomotor, descrito en las primeras líneas, con respecto a las destrezas, pero también a los procesos. Cada participante ha vivido esta experiencia a su manera, acercándose de diferentes formas al uso de las TIC, tanto así que, en el marco de los Laboratorios Vivos, en los dos municipios han nacido

19 Sobrecallo de Colombia es el seudónimo con el que se conoce a uno de los participantes del proyecto. A continuación, se adjunta el enlace en el que se puede acceder a uno de los productos audiovisuales de este participante: https://www.youtube.com/ watch? $v=$ IVMCQLjuiqm\& $=62 \mathrm{~s}$. 
iniciativas como Dancing Kings y el Colectivo de Comunicaciones de Clemencia $+\mathrm{aCtitud},{ }^{20}$ que dan cuenta de la combinación de los tres dominios de aprendizajes.

\section{Impactos en la dimensión afectiva}

Los impactos de la dimensión afectiva fueron entendidos y enmarcados dentro del reconocimiento que tienen las personas de sí mismas en el contexto, la imagen propia según Rogers (1954), y su relacionamiento con los demás. Esta dimensión está asociada a ser, en el sentido de ser en el mundo heideggeriano, ${ }^{21}$ es decir, el despliegue de la persona situada en un contexto específico. Para el desarrollo narrativo de esta dimensión, se realiza un guiño a la obra Rayuela, de Julio Cortázar (2013), al tomar los tres segmentos principales del libro para dar estructura a los hallazgos encontrados.

\section{Del lado de acá (lo que creo que soy)}

En esta sección se expondrán los cambios percibidos por los participantes sobre sí mismos. La línea expositiva que se sigue hace uso de la analogía geométrica, que en muchas ocasiones ayuda a darle un poco de orden aparente y control a nuestra vida: del centro al exterior a manera de esfera. En el centro se encuentra lo más íntimo y cercano a ser y a medida que se va hacia afuera se expone lo que aparente y progresivamente se va alejando del individuo y yendo hacia los demás. De esta manera, en el centro y corazón de la esfera, se encuentra cómo los individuos percibieron el efecto del programa en sí mismos para luego exponer el impacto del programa en su relacionamiento con los demás.

El autoconcepto, o imagen propia, se entiende como el juicio que la persona tiene de sí misma; es decir, cómo se percibe. Este constructo es el que la persona siente como más verdadero en relación con lo que realmente es (Rogers, 1954). Esta imagen propia en los participantes del programa tendió a actualizarse. Se observó un aumento de la sensibilidad para percibir el valor propio (un rescate de la identidad propia y comunitaria) y de las cosas, promovido por los contenidos explorados en las sesiones y por el contacto humano. Por contacto humano se entiende el establecimiento de relaciones auténticas, en el sentido yo-tú y yo-ello buberiano. ${ }^{22}$

20 Para ampliar información sobre estas iniciativas, véase las notas de prensa "A ritmo de Dancing Kings" y "+aCtitud Colectivo de comunicaciones" del periódico Noticias Vivas, en http://laboratoriosvivos.com/wp-content/uploads/2017/03/4-edicion-web.pdf.

21 Es un estar arrojado al mundo y hacia sus posibilidades (Heidegger, 2000).

22 Buber (1995) plantea que el hombre existe solo en relación con un otro. Postula que existen diferentes tipos de relaciones de los individuos con el mundo: las yo-tú y las yo-ello. La relación yo-tú buberiana surge a partir del ser y se da entre sujetos sin otra intención más allá que la del encuentro en sí. Por su parte, la relación yo-ello es la que se da entre un sujeto y un objeto y es de corte utilitarista. 
En este sentido, Julia Batista, quien fue parte del perfil Actores sociales, en el municipio de Clemencia, manifiesta lo siguiente: "Aprendí a relacionarme y a socializarme y a valorarme a mí misma y a valorar las pocas cosas que se ven en el medio ambiente y a aquellas personas que para mí eran insignificantes" (Julia Batista, Clemencia, entrevista, 29 de julio del 2016).

El programa de formación permitió expandir el abanico de posibilidades de ser, es decir, fue un escenario para ubicar en una posición diferente a los actores municipales. Por ejemplo, una madre que en su casa barría, trapeaba, cocinaba, durante el programa de formación fue estudiante; su círculo relacional se expandió y su autoconcepción cambió en la medida en que la trascendencia se desenvolvía (ir más allá de sí misma y estar abierta, el transcendens heideggeriano) y aprendía.

Pienso que ha sido un proceso de mejora continua ya que uno viene con una mentalidad de que no estoy haciendo nada en la casa, nada más hacer el aseo, cocinar, comer, dormir, todo monótono, eso no aguanta, mejor voy a estudiar allá para ver qué hago, pero cuando uno llega acá, o sea, y vive y se relaciona con personas. (Sol Rodríguez, María la Baja, entrevista, 31 de agosto del 2016)

Los participantes manifestaron que las herramientas brindadas fueron útiles para desempeñar sus futuras labores: en la escuela, liderando comunidades, en sus trabajos, tal es el caso de la formadora Luz Barrios:

me ayudó a formarme más como persona, me ayudó a ser una mejor docente para yo poderle brindar esas herramientas tan buenas que aprendí aquí a mis padres de familia para que sea multiplicada a los niños y niñas de la comunidad. (Clemencia, entrevista, 8 de septiembre del 2016)

A partir de esta experiencia, se reconocieron como más libres, mas ellos al "perder la pena", "abrirse", "dejar el pánico escénico", "soltarse", tal como manifestaron varios de los participantes. Todo esto posibilitó que descubrieran su voz, hablaran e hicieran, apegándose más a su ser posible.

\section{Del lado de allá (cómo soy con los demás)}

Este acápite se centra en la manera como cambió el relacionamiento con la otredad. En cualquier situación, una de las principales maneras de combatir las atribuciones erradas, los prejuicios, los estereotipos y la discriminación es a través del contacto directo con la fuente. Es así como cohabitar un mismo espacio generó primero comprensión, luego aceptación, e incluso una valoración hacia personas y grupos con cualidades diferentes a las propias. 
Un compañero va a hacer un documental sobre los cantos de vaquería, lindísimo, y todos los días pasa el ganado por mi casa y todos los días cierro la puerta, y me estorba la bulla que ellos hacen. Ahora me doy cuenta que no, que más bien eso hace parte de su trabajo y que más bien es una actividad muy hermosa, y así como esas actividades hay muchas. (Fermina Villamil, María la Baja, entrevista, 13 de febrero de 2017)

Dentro de los Laboratorios Vivos se formaron redes sociales, ${ }^{23}$ comunidades de sentido donde confluyeron capital simbólico, cultural, tecnológico y social que posibilitaron el reforzamiento, la construcción y la deconstrucción de comunidades creativas, entendidas como grupos de personas vinculadas entre sí por una conexión sentimental (Weber, 2005; Mauss, 2007, citados por Molina et al., 2017). Estos espacios se configuraron como un territorio de trascendencia donde se relacionaban y aprendían con y del otro. Así lo manifiesta el joven del grupo Emprendedores, de Clemencia, Luis David Zárate, "porque aquí en el proyecto también aprendimos que no solo es trabajar como nosotros... no es cómo trabajar individualmente siempre, sino cómo trabajar en grupo, cómo colaborar con los demás" (entrevista, 24 de septiembre del 2016).

Se resalta que se dio prelación a la inteligencia comunitaria sobre la individual, se tejieron redes de conocimiento entre ellos, lo que fortaleció conocimientos prácticos y teóricos que fueron replicados en contextos diferentes al de la Casa de la Cultura, por ejemplo los colegios, al "poner a versear" ${ }^{\prime 24}$ a los estudiantes, o permitir que usaran equipos tecnológicos, como las cámaras, dentro de la institución.

Así mismo, se promovió la convivencia y el diálogo intergeneracional $^{25}$ en diferentes actividades del proyecto como en la elaboración de microdocumentales en el módulo TIC y producción audiovisual en donde se rescataron símbolos culturales y legados inmateriales. Estos escenarios, en los que los ubica el programa de formación generaron nuevas dinámicas que los situaron en nuevas posiciones y los empoderaron al aprender, practicar y demostrar sus habilidades. Esto los hace soñar. Soñar lo que pueden llegar a ser y hacer.

23 Para Jordi Jubany (2012) "se puede entender una red social como una estructura de personas en la que los nodos representan individuos o entidades, y éstos establecen diversas formas de relaciones entre ellos" (p. 28).

24 "Poner a versear" se conoce como la construcción de versos con rima. La profesora María Morales manifestó que, en ocasiones, utilizaba este recurso para conectar a los estudiantes con las distintas temáticas que aborda en el aula. Esta expresión fue utilizada por la profesora de la Institución Educativa San José, de Clemencia, en entrevista del 22 de marzo del 2017.

25 Al respecto del tema, véase Ortega y Marín (2017) en Molina, Mendoza, Ortega et al. (2017). 


\section{De otros lados (como quiero ser)}

"De otros lados" se ocupa de los sueños, del poder ser. Florece desde lo posible, que para Heidegger (2000) es aquello cuya esencia reside en el querer y a partir del querer es capaz de pensar. Es como $\operatorname{ser}^{26}$ es trascendens por antonomasia; es decir, la capacidad que tiene ser de abrirse. Aquí se quiere resaltar la oportunidad que brindó el programa de formación al permitirles a los participantes pensarse en condiciones y situaciones alternativas a la actual, de pensar una Clemencia y María la Baja mejor desde el campo del deseo y la posibilidad.

Existen testimonios que ubicaron a los jóvenes en un comienzo como apáticos con los Laboratorios Vivos, y finalizaban con una perspectiva esperanzadora sobre su futuro y su presente, a manera de una reivindicación de sí mismos de cara al futuro. Este fenómeno se desarrolló al trabajar sobre las capacidades humanas y a partir del ejemplo de sus compañeros, que lograron empezar a estudiar una carrera universitaria, $y$ de sus mediadores de los que —en sus palabras—, se llevaron disciplina y responsabilidad. Así lo mencionó Mauro Burgos, técnico de audio de Clemencia: "Los Laboratorios Vivos dejan más actitud y abrieron la mente de los participantes, tener ganas de ser mejor, cambiar y transformar sus vidas. Los Laboratorios Vivos se pueden acabar, pero eso seguirá, porque está en el cerebro de todos" (Clemencia, entrevista, 13 de marzo del 2017).

Los participantes se hicieron conscientes de la riqueza inmaterial y el potencial personal de desarrollo propio y ajeno. Así, había algunos que querían ser los mejores locutores del mundo, otros querían ser periodistas, algunos diseñadores, otros a través de sus iniciativas de microproyecto buscaban involucrar a toda la comunidad en su proyecto. Se comenzó a gestar un sentimiento de comunidad.

Es así como se encontró una iniciativa muy sentida que buscaba reconocer y honrar de manera comunitaria la memoria de aquellos que ya no están. Es una idea que trabaja con la memoria histórica, con el recuerdo de los muertos y su reivindicación. Es cultivo de la memoria para la paz en un municipio como María la Baja, que fue golpeado particularmente por la violencia. Edilberto Sanabria, actor social, cree que el sentimiento de solidaridad comunitaria se rompió con las acciones violentas del conflicto armado y, por tanto, quiere, a través de la reconstrucción del cementerio de Playón y la construcción de un museo de memoria histórica Ilamado Playón Sueña, ser tejedor y artesano de fibras sociales para reparar el entramado comunitario y volver a creer que unidos pueden hacer las cosas. 
Y que a partir de eso que nazca esta nueva forma de tejedor, porque yo me voy a convertir como en un tejedor, y entonces podamos implementar otras cosas más que tengamos que mejorar dentro de la comunidad, porque figúrate, ya si nosotros nos organizamos y cumplimos con lo del cementerio, bueno ahora vamos a agarrar la iglesia, vamos a agarrar el parque, ahora vamos a agarrar esto y para cualquier otra actividad ya sea institucional o local, va a haber esa fuerza unida de la comunidad porque todos vamos a pensar en colectivo y en objetivos propios discutidos y seleccionados por la misma comunidad. (Edilberto Sanabria, María la Baja, entrevista, 28 de julio del 2016)

La principal estrategia de sostenimiento de iniciativas como los Laboratorios Vivos es que la comunidad se empodere y dé continuidad al proceso con los cambios que considere pertinentes. La exhortación realizada al finalizar este acápite es que invertir en el capital humano de los territorios es lo que a largo plazo brindará desarrollo a las regiones y construcción de comunidad.

\section{Conclusiones}

La manera de evaluar comúnmente los impactos de un programa o proyecto es a través de la revisión del cumplimiento o no de los objetivos formulados al principio (en el caso del programa de formación, estos fueron: aprender a conocer, aprender a hacer y aprender a ser). Normalmente las evaluaciones de impacto en el área social se realizan después de la culminación de los proyectos, programas, cursos, etcétera. En este sentido, se quiere resaltar la necesidad de abordar nuevamente en el futuro a las comunidades que fueron parte de esta intervención para corroborar los impactos generados a largo plazo.

Entre los principales logros del programa de formación y del proyecto estuvo el fomento de la innovación social de base cultural, orientada a generar espacios de convivencia, aprovechamiento del tiempo libre, mejoramiento del diálogo y, en gran medida, el esfuerzo por sentar unas bases para hacer de la cultura un motor de desarrollo humano a escala local, a través de la generación de capacidades en cuanto a la formación de personas de diferentes perfiles y generaciones con mejores condiciones actitudinales y aptitudinales, así como la generación de una capacidad instalada disponible para el aprovechamiento de la comunidad en general.

Parece ser que los Laboratorios Vivos se constituyeron en una experiencia de inclusión social y productiva para los municipios, la cual, mediante la generación de competencias en emprendimiento, llevó a los participantes involucrados a apropiarse de sus ideas y confiar en sus capacidades, dejando en la comunidad a personas con nociones sobre temas de cultura, emprendimiento y TIC. 
Los objetivos del programa de formación tenían puntos de encuentro entre ellos. Gracias a la apuesta pedagógica del programa y a los retos que implicó llevarlo a cabo (por dificultades presentes en el día a día con los participantes), se logró que ellos fueran el centro y la razón de ser de este proyecto, reconocieran sus saberes, valoraran la materia prima con la que cuentan sus municipios, y aprendieran nuevas herramientas y conocimientos que se espera ayuden a disminuir de alguna manera la brecha entre quienes los poseen y quienes no, y construyeran nuevos lazos entre sus coterráneos.

Como autores, interpelamos la manera como se desarrolló la conceptualización de los participantes en el proyecto, en la medida en que fueron concebidos y divididos como seres con tres dimensiones claras y definidas (conocer, hacer y ser). En el desarrollo de la investigación se halló que estas dimensiones son móviles, solubles y con fronteras que en ocasiones se tienden a difuminar. Es por esto que se aboga por una comprensión más holística e integral de aquellas personas que son la sangre del corazón del proyecto. Esto va en consonancia con posturas como las de Darder y Bach (2007) y Darder et al. (2017), en las cuales la integración de pensamiento, emoción y acción son parte de la condición humana y, por lo tanto, deben ser ejes de la acción educativa.

Se considera que iniciativas que invierten recursos físicos, económicos y humanos en la generación de capacidades influirán en el desarrollo del país debido a que a raíz de esta experiencia se observó que la innovación social de base cultural puede ayudar a resolver algunas necesidades que el Estado no ha logrado atender eficazmente, sin que esto justifique su ausencia, Aquí se buscó empoderar a los participantes con conocimientos y herramientas que pudieran replicar en sus comunidades. Fue así como jóvenes de estos municipios decidieron desarrollar iniciativas para mitigar problemas presentes en sus contextos a partir de ideas creativas e innovadoras que han Ilegado a impactar de diferentes formas a las comunidades desde los niveles más micro.

Es así como el programa de formación y los procesos adelantados en los Laboratorios Vivos han representado una oportunidad de aprendizaje, tanto para las comunidades participantes como para el equipo del área de formación y del proyecto, ya que de una u otra manera se constituyó en un escenario propicio para el diálogo de saberes y el intercambio bidireccional de conocimientos. Allí, mediante el enfoque de capacidades cognoscitivas, psicomotoras y afectivas, se desarrollaron competencias para la vida.

\section{Recomendaciones}

Como se puede evidenciar en los resultados de la investigación, los objetivos planteados por el programa de formación se cumplieron de manera general, teniendo en cuenta el alcance y las diferencias de cada uno de los 
perfiles de los participantes, así como sus distintas actitudes, aptitudes y su desempeño en el programa. En ellos se generaron algunos aprendizajes, fundamentalmente en aspectos personales, sociales y culturales, más que en conocimientos técnico-científicos, pero igualmente valiosos. Quizás esto se deba a la imposibilidad de contar con una mayor duración en horas para el desarrollo de los módulos, aspecto que se convierte en uno de los puntos para tener en cuenta en experiencias similares.

Para una futura réplica se pone sobre la mesa la posibilidad de implementar un modelo pedagógico más contextualizado para este tipo de comunidades, que comprenda los diferentes dominios de aprendizaje de manera integral, y que sean vistos como un todo. Para ello es importante que en la planeación del programa de formación y sus componentes, se tenga en cuenta un conocimiento previo de los municipios y sus habitantes para tener una mejor visión de las comunidades que se van a intervenir con este tipo de iniciativas. Esto permitiría repensar qué otras temáticas sería pertinente y relevante desarrollar.

Si bien algunos participantes aprendieron contenidos de los módulos, se apropiaron de ellos y los aplicaron, otros no encontraron, en principio, que lo visto en el programa resultara relevante para su vida, aspecto vertebral del aprendizaje significativo. Sin embargo, se espera que lo aprendido, de una u otra manera, haya dejado reflexiones que ayudaran en su cotidianidad.

El fin último de este proyecto va más allá de una apropiación de conocimientos técnicos, culturales o sociales, se direcciona a la construcción de ciudadanía y al aumento de la calidad de vida de los habitantes de los municipios de Clemencia y María la Baja. Esto se traduce en que son muchos más los aprendizajes adquiridos relacionados, por ejemplo, con el uso del tiempo libre, fomento de ideas nuevas y proyectos personales o colectivos, descubrimiento de habilidades, aumento de la autoestima, y reafirmación de las identidades individuales y grupales. Este proyecto fue un detonante que abrió infinitas posibilidades para los participantes que supieron aprovechar esta oportunidad, que apenas empiezan a descubrir sus capacidades de visionar en grande, por y para ellos y sus comunidades, ampliando así sus perspectivas. Se reitera que es importante poder realizar estudios sobre los impactos causados a largo plazo.

\section{Referencias}

Almeida, G. (2005). El constructivismo como modelo pedagógico. http:// galeon.com/tributaria1/Documentos/Constructivismo.pdf.

Ausubel, D. (1983). Psicología educativa. Un punto de vista cognoscitivo (2. ${ }^{a}$ ed.). Trillas. 
Ausubel, D. (2002). Adquisición y retención del conocimiento: una perspectiva cognitiva. Ediciones Paidós Ibérica.

Bello, R. (2009). Evaluación de impacto. http://www.cepal.org/ilpes/noticias/paginas/9/37779/impacto_rbbcproy.pdf.

Buber, M. (1995). Yo y tú. Caparrós.

Chaparro, F. (2001). Conocimiento, aprendizaje y capital social como motor de desarrollo. Ci. Inf., Brasília, 30, 19-31.

Cortázar, J. (2013). Rayuela. Alfaguara.

Coll, C., Martín, E. Mauri, T., Miras, M., Onrubia, J., Solé, I. y Zabala, A. (1997). El constructivismo en el aula. Editorial Graó.

Darder, P. y Bach, E. (2007). Aportaciones para repensar la teoría y la práctica educativas desde las emociones. Teoría de la Educación. Revista Interuniversitaria 18, 55-84.

Darder, P., Salmurri, F., Royo, M., Carpena, A., Sala, J., Marzo, L. y Albadalejo, M. (2017). La formación emocional del profesorado. Aprender y educar con bienestar y empatía. Octaedro.

Gergen, K. (1996). Realidades y relaciones. Aproximaciones a la construcción social. Paidós.

Gobernación de Bolívar, Instituto de Cultura y Turismo de Bolívar (Icultur) y Universidad de Bogotá Jorge Tadeo Lozano Seccional del Caribe (Utadeo). (2014). Implementación de una estrategia para el uso y apropiación de la Cultura como generadora de conocimiento e innovación social, a través de laboratorios sociales de investigación y creación en el departamento de Bolívar. [Documento técnico del proyecto]. Documentos de Circulación Interna del Proyecto Laboratorios Vivos de Innovación y Cultura. Archivo documental de la Fundación Universidad de Bogotá Jorge Tadeo Lozano, Seccional del Caribe, Cartagena de Indias.

Gobierno de Colombia. (2009). Ley 1341, de 30 de julio de 2009por la cual se definen principios y conceptos sobre la sociedad de la información y la organización de las tecnologías de la información y las comunicaciones (TIC), se crea la Agencia Nacional de Espectro y se dictan otras disposiciones. Ministerio de Tecnologías de la Información y las Comunicaciones.

Harrow, A. (1978). Taxonomía del dominio psicomotor. El Ateneo.

Heidegger, M. (2000). Carta sobre el humanismo. Alianza.

Jubany, J. (2012). Aprendizaje social y personalizado: conectarse para aprender. Editorial voc.

Laboratorios Vivos de Innovación y Cultura. (2017, marzo). A ritmo de Dancing Kings, Noticias Vivas. http://laboratoriosvivos.com/wp-content/uploads/2017/03/4-edicion-web.pdf. 
Laboratorios Vivos de Innovación y Cultura. (2017, marzo). +aCtitud Colectivo de Comunicaciones, Noticias Vivas. http://laboratoriosvivos. com/wp-content/uploads/2017/03/4-edicion-web.pdf.

Medina, M. y Verdejo, A. (2001). Evaluación del aprendizaje estudiantil. Isla Negra.

Miranda, J. (2004). El aprendizaje escolar y la metáfora de la construcción. http://www. odiseo.com.mx/2004/01/colaboradores.htm.

Molina, G., Zúñiga, M., Mendoza, L. y Marín, K. (2017). Introducción: al encuentro de una microrrevolución creativa en el norte de Colombia. En G. Molina, L. Mendoza, A. Ortega, F. Ochoa, M. Barraza, ... M. Zúñiga, Tecnologías simbólicas y culturas creativas. La experiencia regional de los Laboratorios Vivos de Innovación y Cultura (pp. 15-39). Universidad de Bogotá Jorge Tadeo Lozano.

Ortega, A. y Marín, K. (2017). Primeros pasos en materia tecnológica: caracterización del estado del uso de las tIC en los municipios de Clemencia y María la Baja. En G. Molina, L. Mendoza, A. Ortega, F. Ochoa, M. Barraza, ... M. Zúñiga, Tecnologías simbólicas y culturas creativas. La experiencia regional de los Laboratorios Vivos de Innovación y Cultura (pp. 129-135). Universidad de Bogotá Jorge Tadeo Lozano.

Osorio, D. y García, M. (2017). Imagen-espacio de los territorios locales: Clemencia y María la Baja. En G. Molina, L. Mendoza, A. Ortega, F. Ochoa, M. Barraza, ... M. Zúñiga, Tecnologías simbólicas y culturas creativas. La experiencia regional de los Laboratorios Vivos de Innovación y Cultura (pp. 44-74). Universidad de Bogotá Jorge Tadeo Lozano.

Programa de las Naciones Unidas (PNUD). (2016). Informe regional sobre Desarrollo Humano para América Latina y el Caribe. http://www. co.undp.org/content/dam/colombia/docs/DesarrolloHumano/ undp-co-idhreview-2016.pdf?download.

Ramírez, A. (1999). El constructivismo pedagógico. http://ww2.educarchile.cl/UserFiles/P0001\%5C\%5CFile\%5C\%5cEl\%20Constructivismo\%20Pedagógico.pdf

Rogers, C. (1954). Psycho-therapy and personality change. University of Chicago Press.

Sen, A. (2004). ¿Cómo importa la cultura en el desarrollo? Revista Diversidad Cultural, Desarrollo y Cohesión Social, 1, 78-94.

Torres, A. (2006). Aprendizaje basado en la investigación. Técnicas didácticas. http://www.itesca.edu.mx/documentos/desarrollo_academico/ Metodo_Aprendizaje_Basado_en_Investigacion.pdf.

Universidad de Bogotá Jorge Tadeo Lozano Seccional del Caribe (Utadeo). (2016). Manual proyecto educativo del Programa de Formación con Enfoque de Innovación Social. Publiday. 